\title{
Human homologue for the mouse mutant disorganisation: does it exist?
}

\author{
K K Naguib, M S Hamoud, E S Khalil, M Y El-Khalifa
}

\begin{abstract}
We describe a newborn Arab male with defects similar to those seen in mice heterozygous for the mutant disorganisation (DS) gene. He had complete absence of the left lower limb including the left pelvic bone, hamartomas arising from the abdominal wall, a small penis, absent left half of the scrotal sac, absent left testicle, anterior displacement of the anus, and multiple vertebral defects. The similarity between the proband's anomalies and those found in affected heterozygotes for DS support the possibility of a human homologue of the DS gene.
\end{abstract}

The mouse mutant disorganisation (DS) gene has been described by Hummel. ${ }^{1}$ It is a semidominant gene with variable expressivity and penetrance, resulting in several abnormalities. Recently the possibility of human homologues for the DS gene was raised. ${ }^{2-3}$ Here we present an Arab male neonate who has similar features to the mouse mutant.

\section{Case report}

The proband, a Syrian male, was the product of the third pregnancy of non-consanguineous, phenotypically normal, young parents. Pregnancy and delivery were uneventful, and spontaneous vaginal delivery occurred at term. Another two pregnancies ended in early spontaneous abortion. On examination his weight was $2500 \mathrm{~g}$, length $48 \mathrm{~cm}$, and OFC 31.5 $\mathrm{cm}$. There was complete absence of the left lower limb, two small papular skin appendages arising from

Kuwait Medical Genetics Centre, Maternity Hospital, PO Box 4080, Safat 13041, Kuwait.

K K Naguib, M Y El-Khalifa

Neonatology Unit, Maternity Hospital, Kuwait. M S Hamoud

Department of Paediatrics, Al-Sabah Hospital, Kuwait. E S Khalil

Correspondence to Dr Naguib.

Received for publication 28 June 1990

Accepted for publication 7 September 1990. the abdominal wall in the suprapubic region, a cystic swelling overlapped by telangiectatic skin on the left loin, a small penis, and absent left half of the scrotal sac and left testis. There was also a right inguinal hernia, a right hydrocele, herniation in the left lower abdominal wall owing to a muscular defect, deep abnormal plantar creases, and anterior displacement of the anus. There was no evidence of amniotic bands. Other body parts including craniofacial features were normal. Skeletal survey showed an absent left lower limb including the left pelvic bones and multiple hemivertebrae. The sacral and lumbar spine was slightly bowed with convexity to the right, and the sacrum appeared short. The lumbar vertebrae and the sacral segments were malformed showing several block vertebrae and wide open neural arches. MCUG showed that the bladder was situated in the right half of the pelvis and was of small capacity (about 15 to 20 $c c)$. The duration of micturition was short. The urethra remained filled with some dribbling after emptying and there was bladder residue (probably neuropathic). Abdominal ultrasound showed a normal echogenic pattern of liver parenchyma, gall bladder, and spleen. The right kidney was of normal size and showed hyperechogenicity and DMSA scan confirmed solitary renal tissue in the right lower abdomen. Renal dynamic study showed an adequately functioning right kidney with no evidence of obstruction, but no left kidney was identified.

\section{Discussion}

The mouse mutant disorganisation (DS) gene was first described by Hummel. ${ }^{1}$ It is a semidominant gene with variable penetrance and expressivity. It is lethal when present in the homozygous state. In affected heterozygotes, $72 \%$ will manifest malformations; two thirds of them have multiple defects and the rest have single defects. The anomalies and their similarity to human malformation patterns have recently been reviewed. ${ }^{23}$

In the present report, the proband has many features similar to those seen in mice heterozygous for DS: complete absence of the left lower limb, absent left scrotal sac and left testicle, papular skin appendages arising from the lower abdominal wall in the suprapubic region, and absent left kidney. 
Alternatively, these anomalies may represent a form of limb-body wall complex (LBWC) without the abdominal defect resulting from either vascular disruption or mechanical compression. ${ }^{45}$ These structural defects are thought to be the result of early amnion rupture and the different combination of anomalies described depends on the time at which the event took place. ${ }^{6}$ However, it seems unlikely that the malformations present in the proband could be explained by amniotic band disruption because of the lack of abdominal wall defect with evisceration of the internal organs, which are essential for the diagnosis of LBWC. ${ }^{7}$

Recently, the hypothesis of an intrinsic defect of germ plasma to explain the abnormalities observed in amniotic band sequences, which was rejected by Torpin, ${ }^{8}$ has been raised again in several reports. ${ }^{2}{ }^{3}$ The similarity between the anomalies resulting from LBWC and affected heterozygous DS raises the possibility that both conditions represent one entity characterised by a wide range of expressivity and penetrance.
Our case supports the existence of a human homologue of the DS gene suggested by Winter and Donnai. $^{2}$

The authors thank Mrs Regina Ratos for typing the manuscript.

1 Hummel KP. The inheritance and expression of disorganization, an unusual mutation in the mouse. $\mathcal{F}$ Exp Zool 1958;137: $389-423$.

2 Winter R, Donnai D. A possible human homologue for the mouse mutant disorganisation. $\mathcal{J}$ Med Genet 1989;26:417-20.

3 Donnai D, Winter R. Disorganisation: a model for 'early amnion rupture'? F Med Genet 1989;26:421-5.

4 Van Allen $M$. Fetal vascular disruptions: mechanisms and some resulting birth defects. Pediatr Ann 1981;10:219-33.

5 Miller ME. Structural defects as a consequence of early intrauterine constraint: limb deficiency, polydactyly and body wall defects. Semin Perinatol 1983;7:274-7.

6 Smith DW. Recognizable patterns of human malformations. 3rd ed. Philadelphia: Saunders, 1982: 486-96.

7 Litwin A, Merlob P, Grunebaum M. Complete absence of external genitalia in limb-body wall complex: two cases. $\mathcal{F}$ Med Genet 1988;25:340-3.

8 Torpin R. Amniochorionic mesoblastic fibrous strings and amniotic bands. Associated constricting fetal malformations or fetal death. Am f Obstet Gynecol 1965;91:65-75. 